\title{
Modeling and Management of Nonlinear Dependencies-Copulas in Dynamic Financial ANALYSIS
}

\author{
Martin Eling \\ Denis Toplek
}

\begin{abstract}
We study the influence of nonlinear dependencies on a non-life insurer's risk and return profile. To achieve this, we integrate several copula models in a dynamic financial analysis framework and conduct numerical tests. We also test risk management strategies in response to adverse outcomes. Nonlinear dependencies have a crucial influence on the insurer's risk profile that can hardly be affected by the analyzed management strategies. We find large differences in risk assessment for the ruin probability and for the expected policyholder deficit. This has important implications for insurers, regulators, and rating agencies that use these measures as a foundation for internal risk models, capital standards, and ratings.
\end{abstract}

\section{INTRODUCTION}

Dynamic financial analysis (DFA) is a financial modeling approach that projects financial results under a variety of possible scenarios, showing how outcomes might be affected by changing internal and external conditions (see Casualty Actuarial Society, 2006). DFA has become an important tool for decision making and an essential part of enterprise risk management (ERM), particularly within the field of non-life insurance. The DFA results and the quality of decisions derived from them depend on an appropriate modeling of the stochastic behavior of assets and liabilities. In this context, the correct mapping of nonlinear dependencies is of central concern. Although

Martin Eling is with the Institute of Insurance Science, Ulm University, Ulm, Germany. Denis Toplek is with the Institute of Insurance Economics, University of St. Gallen, St. Gallen, Switzerland. The authors can be contacted via e-mail: martin.eling@uni-ulm.de and denis.toplek @unisg.ch. The authors are grateful to anonymous referees, Andrew Cairns, Paul Embrechts, Edward Frees, Nadine Gatzert, Gudrun Hoermann, Steffi Höse, Thomas Parnitzke, Hato Schmeiser, Joan T. Schmit, and Gary Venter; the participants of the 14th Annual Meeting of the German Finance Association, Dresden; the 11th International Congress on Insurance: Mathematics and Economics, Piraeus; the 37th ASTIN Colloquium, Orlando; the 16th International AFIR Colloquium, Stockholm; the 7th German Operations Research Society Workshop on Finance, Augsburg; and the Research Seminar in Actuarial Science, Risk Management, and Insurance at the University of Wisconsin-Madison for valuable suggestions and comments. 
many DFA models and most practitioners still focus on linear correlation, the literature suggests that solely considering linear correlation is not appropriate in modeling dependence structures between heavy-tailed and skewed risks, which are frequent in the insurance context (see, e.g., Embrechts, McNeil, and Straumann, 2002). These risks are especially relevant in case of extreme events, e.g., the September 11, 2001, terrorist attacks that resulted in insurance companies experiencing large losses both from their underwriting business and the related capital markets plunge (see, e.g., Achleitner, Biebel, and Wichels, 2002). A more recent example is the subprime credit crisis, in which insurers have sustained large losses from their investments, e.g., in mortgage-backed securities, as well as from insuring structured credit products such as collateralized debt obligations (American International Group (AIG) is the most prominent example).

In this article, we evaluate the influence of such extreme events on a nonlife insurer's risk and return profile. We integrate nonlinear dependencies in a DFA framework using the copulas concept and evaluate their effects on the insurer's risk and return distribution within a simulation study. As one cannot generally say which copula describes reality best, we compare different forms of copulas (i.e., the Gauss, $t$, Gumbel, Clayton, and Frank copulas) and evaluate the possible impact in a stress-testing sense.

In our simulation study, we find that nonlinear dependencies have a strong influence on the insurer's default risk and performance. We also find different impacts of nonlinear dependencies on ruin probability and expected policyholder deficit, a result that is of special relevance for policyholders, regulators, and rating agencies. For example, for some kinds of nonlinear dependencies, the expected policyholder deficit cannot be reduced by increasing equity capital. It thus seems that these tail dependencies are relevant not only for low-capitalized companies but also for well-capitalized ones. Furthermore, we test several risk management strategies implemented in response to adverse outcomes generated by nonlinear dependencies. Our simulation results show that simple risk-reduction strategies are of little use. For example, a reinsurance strategy can delimit the high ruin probability generated by nonlinear dependencies, but not necessarily the expected policyholder deficit.

Our article builds upon two branches of literature-DFA and the copulas concept. In the late 1990s, the Casualty Actuarial Society introduced simulation models for property-casualty insurers, calling them "DFA" (see Casualty Actuarial Society, 2006). Since then, several surveys and applications of DFA have been published in academic journals. Lowe and Stanard (1997), as well as Kaufmann, Gadmer, and Klett (2001), provide an introduction to DFA by presenting a model framework and an application of their model. Lowe and Stanard develop a DFA model for the underwriting, investment, and capital management process of a property-catastrophe reinsurer and Kaufmann, Gadmer, and Klett provide an up-and-running model for a nonlife insurance company. Blum et al. (2001), D'Arcy and Gorvett (2004), and Eling, Parnitzke, and Schmeiser (2008) use DFA to examine specific decision making situations. Blum et al. investigate the impact of foreign-exchange risks on reinsurance decisions within a DFA framework and D'Arcy and Gorvett apply DFA to search for an optimal growth rate in the property-casualty insurance business. The influence of 
management strategies on an insurer's risk and return position using DFA is investigated in Eling, Parnitzke, and Schmeiser.

The copulas concept and the problem of mapping nonlinear dependencies in an insurance context was first introduced by Wang (1998), who discusses models and algorithms for the aggregation of correlated risk portfolios. Frees and Valdez (1998) also provide an introduction to the use of copulas in risk measurement by describing the basic properties of copulas, their relationships to measures of dependence, and several families of copulas. Klugman and Parsa (1999), Mashal and Zeevi (2002), Malevergne and Sornette (2003), Dias (2004), and Kole, Koedijk, and Verbeek (2007) develop appropriate models to analyze capital and insurance markets by fitting copulas to empirical data. Blum, Dias, and Embrechts (2002) discuss the use of copulas to handle the measurement of dependence in alternative risk transfer products. Embrechts, McNeil, and Straumann (2002) present properties, pitfalls, and simulation algorithms for correlation and dependence in risk management and analyze the effect of dependence structures on the value at risk. Pfeifer and Nešlehová (2004) propose approaches for modeling and generating dependent risk processes in the framework of collective risk theory. McNeil (2007) presents algorithms for sampling from a specific copula class which can be used for higher-dimensional problems.

The aim of this article is to contribute to this literature by integrating copulas in an extended version of the DFA model presented by Eling, Parnitzke, and Schmeiser (2008) and by evaluating their influence on the insurer's risk and return position. Our results indicate that it is crucial to consider the copulas concept in order to improve DFA and decision making in ERM of insurance and reinsurance companies. Our findings are also important for regulators and rating agencies as they reveal large differences in risk assessment for the expected policyholder deficit and for the ruin probability. As these measures are the basis of many capital standards and ratings, it is important to integrate nonlinear dependencies in the regulatory framework and in rating assessment, e.g., in stress testing and scenario analysis. ${ }^{1}$ Furthermore, these measures are the foundation of internal risk models emphasizing the relevance of the results for insurers and reinsurers.

The rest of the article is organized as follows. In the "Model Framework" section we present a DFA framework containing the essential elements of a nonlife insurance company. In the "Integration of Copulas in DFA" section we describe the copulas concept, different types of copulas, and how we integrated them in the DFA framework. In the "Measurement of Risk, Return, and Performance in DFA" section we define financial ratios, reflecting both risk and return in a DFA context. A DFA simulation

\footnotetext{
${ }^{1}$ Ibragimov and Walden (2007) and Ibragimov, Jaffee, and Walden (in press) investigate the diversity of optimal insurance in the presence of heavy tails versus light tails. Although these two studies analyze individual risks, our focus is on enterprise risk management at the wholecompany level. Ibragimov, Jaffee, and Walden analyze i.i.d. risks and Ibragimov and Walden symmetric spherical distributed risks, whereas we consider the dependence of differently distributed risks including nonlinear and, in particular, asymmetric dependence. Our model not only addresses value at risk, but also looks at several other risk measures, return measures, and the risk-adjusted performance of the company. Ibragimov, Jaffee, and Walden; Ibragimov and Walden; and our paper highlight the fact that solely considering linear correlation is not appropriate in modeling dependence structures between heavy-tailed and skewed risks.
} 
study to examine the effects of the copulas on risk and return is presented in the "Measuring the Influence of Copulas in DFA" section. In the "Measuring the Influence of Copulas in DFA" section we measure the influence of risk management strategies implemented as a response to adverse outcomes generated by the copulas. And the final section concludes.

\section{MOdel FramewOrK}

We build on the DFA framework used by Eling, Parnitzke, and Schmeiser (2008) to investigate the influence of management strategies on an insurer's risk and return. In this model, a management rule changes the portion of risky investments and the market share in the underwriting business depending on the insurer's financial situation. We extend this framework with a modified underwriting cycle following an autoregressive process and a modified claims process consisting of noncatastrophe and catastrophe losses.

Let $E C_{t}$ be the equity capital of the insurance company at the end of time period $t$ ( $t \in$ $1, \ldots, T)$ and $E_{t}$ the company's earnings in $t$. Then, development of the equity capital over time can be written as

$$
E C_{t}=E C_{t-1}+E_{t}
$$

The financial statement earnings $E_{t}$ in period $t$ consist of the investment result $I_{t}$ and the underwriting result $U_{t}$. In case of positive earnings, taxes are paid. We denote $t r$ as the tax rate and obtain the company's earnings in $t$ as

$$
E_{t}=I_{t}+U_{t}-\max \left(t r \cdot\left(I_{t}+U_{t}\right), 0\right)
$$

The assets can be divided in high-risk investments, such as stocks or high-yield bonds, and low-risk investments, such as government bonds or money market instruments. We denote $\alpha_{t-1}$ as the portion of high-risk investments in time period $t$ and $r_{1 t}\left(r_{2 t}\right)$ as the return of the high-risk (low-risk) investment in $t$. The return of the company's investment portfolio in $t\left(r_{p t}\right)$ can be calculated as

$$
r_{p t}=\alpha_{t-1} \cdot r_{1 t}+\left(1-\alpha_{t-1}\right) \cdot r_{2 t}
$$

By multiplying the portfolio return with the funds available for investments, we calculate the company's investment result. The funds available for investments are the equity capital and the premium income $P_{t-1}$, less upfront costs $E x_{t-1}^{P}$ :

$$
I_{t}=r_{p t}\left(E C_{t-1}+P_{t-1}-E x_{t-1}^{P}\right) .
$$

To model the underwriting business, we denote $\beta_{t-1}$ as the company's portion of the relevant market in $t$. The underwriting market accessible to the insurer (given by $M V$ ) is obtained with $\beta_{t-1}=1$. The market volume rises by the rate $i$, which might represent average market growth or compensate for inflation. The premium rate level 
achievable in the market has been observed to exhibit a cyclical pattern. Following Cummins and Outreville (1987), we model the underwriting cycle using a stationary autoregressive process of order two (with the parameters $a_{0}, a_{1}$, and $a_{2}$ for lags 0,1 , and 2):

$$
\Pi_{t}=a_{0}+a_{1} \Pi_{t-1}+a_{2} \Pi_{t-2}+\varepsilon_{t}
$$

The rate level $\Pi_{t}$ depends on the premium levels of the two previous periods and a random error term $\varepsilon_{t}$ following a white noise process. Depending on the parameterization, the process produces cycle lengths that can be calibrated according to observed data. The premium income $P_{t}$ in period $t$ thus depends on the premium rate level $\Pi_{t} .^{2}$

Based on an experiment, Wakker, Thaler, and Tversky (1997) showed that a rise in default risk leads to a rapid decline in the achievable premium level. The premium income should thus not only be connected to the underwriting cycle but also to a consumer response function. The consumer response function (denoted by the parameter $\mathrm{cr}$ ) represents the link between the premiums written and the company's safety level. We determine the safety level by considering the equity capital at the end of the previous period. The premium income in our model is given as

$$
P_{t-1}=c r_{t-1}^{E C_{t-1}} \cdot \Pi_{t-1} \cdot \beta_{t-1} \cdot M V \cdot(1+i)^{t-1} \text {. }
$$

Two types of costs are integrated in the model: upfront costs $\left(E x_{t-1}^{P}\right)$ and claim settlement costs $\left(E x_{t}^{C}\right)$. The upfront costs depend linearly on the level of written market volume (modeled with the factor $\gamma$ ), and quadratically on the change in written market volume (modeled with the factor $\eta$, e.g., because of increased advertising and promotion efforts). The upfront costs $E x_{t-1}^{P}$ are thus calculated as

$$
E x_{t-1}^{P}=\gamma \cdot \beta_{t-1} \cdot M V \cdot(1+i)^{t-1}+\eta \cdot\left(\left(\beta_{t-1}-\beta_{t-2}\right) \cdot M V \cdot(1+i)^{t-1}\right)^{2} .
$$

Claim settlement costs are given as a portion $\delta$ of the claims (denoted by $C$ ) incurred $\left(E x_{t}^{C}=\delta C_{t}\right)$. The claims consist of noncatastrophe losses and catastrophe losses $(C=$ $\left.C_{n c a t}+C_{c a t}\right)$. The underwriting result is thus given by:

$$
U_{t}=P_{t-1}-C_{t}-E x_{t-1}^{P}-E x_{t}^{C} .
$$

\footnotetext{
${ }^{2}$ The presented autoregressive process is the most widespread approach to model the cyclical pattern of premium rate level over time; see Venezian (1985), Niehaus and Terry (1993), Daykin, Pentikäinen, and Pesonen (1994), Lamm-Tennant and Weiss (1997), Fung et al. (1998), Chen, Wong, and Lee (1999), and Meier (2006) for an overview. An alternative in the context of DFA is to use Markov processes and transition probabilities as done in Eling, Parnitzke, and Schmeiser (2008) and D'Arcy et al. (1998). We implemented this approach as a robustness test and found that this modeling alternative does not influence our main results.
} 
Although we restrict ourselves to the standard components necessary in DFA (see Kaufmann, Gadmer, and Klett, 2001, p. 218), one might argue that the model depends on many parameters. However, there is a trade-off between accuracy and complexity. Our aim is to take a holistic view of the company's assets and liabilities and we include the essential elements of a nonlife insurance company and calibrate them using realistic data. Within this setting, we isolate the effect of different dependencies on risk and return. We present results for alternative copulas and parameter settings in order to assess the range of possible outcomes under different scenarios. For the sake of clarity, a table containing all model parameters (and their initial values) is presented in the "Model Specifications" section.

\section{INTEGRATION OF COPULAS IN DFA}

In this section, we consider the modeling of dependencies between risk categories, i.e., between different asset classes (high-risk versus low-risk investments), different kinds of liabilities (noncatastrophe losses versus catastrophe losses), and between assets and liabilities. The dependencies between these risk categories can be integrated in DFA by generating correlated random numbers.

As the literature suggests that solely considering linear correlation is not appropriate in modeling dependence structures between heavy-tailed and skewed risks, we use copulas to model nonlinear dependencies and a rank correlation measure (Kendall's tau) that is invariant under monotonic transformations and thus not affected by the marginal distributions (see, e.g., McNeil, Frey, and Embrechts, 2005, pp. 206-208). Copulas provide a means of separating the description of a dependence structure from the marginal distributions (see Embrechts, 2009, for an overview of the copulas concept).

To investigate the effects of different copulas, we model correlations between highrisk investments, low-risk investments, noncatastrophe losses, and catastrophe losses using the Gauss copula, the $t$ copula, and three nonexchangeable Archimedean copulas (Gumbel, Clayton, Frank). We selected these copulas because they are actually used in practice, easy to parameterize, and simple to calibrate from a technical perspective (see, e.g., Sun, Frees, and Rosenberg, 2008; SCOR Switzerland AG, 2008). In addition, they exhibit different forms of tail dependence and thus are useful for scenario analyses and stress testing. ${ }^{3,4}$ Also useful in the context of scenario analysis

\footnotetext{
${ }^{3}$ According to Joe (1997, p. 33) tail dependence can be defined as follows: $\lambda_{L}=\lim _{u \rightarrow 0+} \operatorname{Pr}\left(U_{1} \leq\right.$ $u \mid U_{2} \leq u$. $)=\lim _{u \rightarrow 0+} \operatorname{Pr}\left(U_{2} \leq u, U_{1} \leq u\right) / \operatorname{Pr}\left(U_{2} \leq u\right)$ is the lower tail dependence parameter for two standard uniform random variables $U_{1}, U_{2}$ with joint distribution function $C\left(U_{1}, U_{2}\right)$. Upper tail dependence $\lambda_{U}$ can be defined analogously. Note that there are different definitions of tail dependence measures in the literature that can lead to different indications of asymptotic dependence and independence; see, e.g., Coles, Heffernan, and Tawn (1999) and Charpentier (2006). Tail dependence is a copula property as the tail dependence coefficient can be expressed in terms of the copula; see, e.g., McNeil, Frey, and Embrechts (2005, p. 209).

${ }^{4}$ An empirical motivation for selection of the copulas would be ideal, but it is problematic to calibrate parametric copulas to aggregated empirical data because the volume of data is rarely sufficient. Copula selection and calibration methods are discussed in the literature (see, e.g., Genest and Rivest, 1993; Chen and Fan, 2006; Patton, 2006). An important argument for selecting the Clayton copula is that it provides a natural limit for conditional bivariate
} 
is that all these copulas span the full range from bivariate countermonotonicity to comonotonicity (except for the Gumbel, which only ranges from independence to comonotonicity).

The Gauss and the $t$ copulas have been studied extensively in risk management literature (see, e.g., McNeil, Frey, and Embrechts, 2005). The Gauss copula is contained in the multivariate normal distribution and does not exhibit tail dependence:

$$
C_{P}^{\text {Gauss }}(\mathbf{u})=\boldsymbol{\Phi}_{P}\left(\Phi^{-1}\left(u_{1}\right), \Phi^{-1}\left(u_{2}\right), \Phi^{-1}\left(u_{3}\right), \Phi^{-1}\left(u_{4}\right)\right)
$$

And $\Phi$ denotes the standard univariate normal density function and $\boldsymbol{\Phi}_{P}$ is the joint density function of a four-dimensional Gaussian vector $\mathbf{u}$ with correlation matrix $P$. The $t$ copula arises from the multivariate Student- $t$ distribution. In contrast to the Gauss copula, the $t$ copula exhibits upper and lower tail dependence (see Demarta and McNeil, 2005).

$$
C_{v, P}^{t}(\mathbf{u})=\mathbf{t}_{v, P}\left(t_{v}^{-1}\left(u_{1}\right), t_{v}^{-1}\left(u_{2}\right), t_{v}^{-1}\left(u_{3}\right), t_{v}^{-1}\left(u_{4}\right)\right),
$$

where $t_{v}$ is the density function of a standard univariate $t$ distribution and $\mathbf{t}_{v, P}$ is the joint density function of a four-dimensional vector with correlation matrix $P$. The subscript $v$ denotes the degrees of freedom of the multivariate $t$ distribution.

McNeil, Frey, and Embrechts (2005) propose a method to calibrate elliptical copulas such as the Gauss and the $t$ copula using the relationship between Kendall's rank correlation $\rho_{\tau}$ and the off-diagonal elements $\rho_{i j}$ of the correlation matrix $P$, where $\rho_{i j}$ stands for the correlation between the two random variables $X_{i}$ and $X_{j}$. We follow their approach and calibrate the Gauss and the $t$ copula parameters according to

$$
\rho_{\tau}\left(X_{i}, X_{j}\right)=(2 / \pi) \arcsin \rho_{i j}
$$

In addition to the Gauss and $t$ copulas, we implement three Archimedean copulas and their respective Survival copulas. The key characteristic of Archimedean copulas is that they can be easily constructed using generator functions $\phi(u)$ (see Nelsen, 2006, p. 109). We use three different copulas to account for different types of tail dependence. The Gumbel copula shows upper tail dependence, the Clayton copula has dependence in the lower tail, and the Frank copula exhibits no tail dependence. In the insurance industry, a threatening development is simultaneous high losses in several lines of business and/or low asset returns in different asset classes. Such a situation could occur, for example, during a financial market crisis where insurers are exposed to financial risk on both the asset and liability sides of the balance sheet. Other adverse scenarios involve natural or man-made catastrophes that lead to high losses and have severe impacts on the financial markets. In our analysis, such developments are visualized by the Clayton copula, which exhibits lower tail dependence (i.e., low

extremes having an Archimedean dependence structure. This is especially relevant for practical purposes when only few data are available and fitting of copulas is not possible (see Juri and Wüthrich, 2002). 
returns in asset classes and low underwriting result corresponding to high losses in several lines of business), and can be thought of as an analysis of the worstcase scenarios in a stress-testing context. Usually, however, data for analyzing tail dependence are rarely available in sufficient volume in most insurance companies. In practice, the form of tail dependence can be deduced based only on available data. However, these can be complemented by scenario analyses of adverse outcomes by employing the Clayton copula.

We also consider the Survival Gumbel and Survival Clayton copulas, which can be generated by transforming the Gumbel and Clayton random numbers with $1-u$. The Survival Gumbel exhibits lower tail dependence and the Survival Clayton upper tail dependence. Note that we do not consider the Survival Frank copula, as it is symmetric and equal to the Survival copula. The generator functions $\phi(u)$ for the five copulas are given in Table 1. $\theta$ denotes the respective copula parameter.

Archimedean copulas can be calibrated to data based on the functional relationship between Kendall's rank correlation $\rho_{\tau}$ and the copula parameter $\theta$. These relationships are summarized in the fourth column of Table 1 for the copulas used in our model. For example, Kendall's tau $\rho_{\tau}$ equals $1-1 / \theta$ for the Gumbel copula. By inverting this relationship, the parameter value $\theta$ can be obtained for any given value of $\rho_{\tau}$.

The family of Archimedean copulas contains both exchangeable and nonexchangeable copulas. As exchangeable copulas impose restrictive conditions on the dependence structure, especially in a multivariate context (e.g., exchangeable copulas result in the same correlation within liabilities as between assets and liabilities), we will use nonexchangeable copulas in order to avoid these unfavorable features. We choose a four-dimensional nonexchangeable construction, described in McNeil, Frey, and Embrechts (2005), consisting of three strict Archimedean generators with completely monotonic inverses and composite functions $\phi_{3} \circ \phi_{1}^{-1}$ and $\phi_{3} \circ \phi_{2}^{-1}$ :

$$
\begin{aligned}
C\left(u_{1}, u_{2}, u_{3}, u_{4}\right)= & \phi_{3}^{-1}(\phi_{3} \circ \underbrace{\phi_{1}^{-1}\left(\phi_{1}\left(u_{1}\right)+\phi_{1}\left(u_{2}\right)\right)}_{\text {high-risk and low-risk investments }}) \\
& +\phi_{3} \circ \underbrace{\phi_{2}^{-1}\left(\phi_{2}\left(u_{3}\right)+\phi_{2}\left(u_{4}\right)\right)}_{\text {noncatastrophe and catastrophe losses }}) .
\end{aligned}
$$

There are other possible four-dimensional nonexchangeable constructions, but this one proves helpful because it results in two exchangeable groups. The first group consists of the high-risk and the low-risk investments and the second group of noncatastrophe losses and catastrophe losses. Thus, we are able to calibrate the copulas according to different correlations for assets and liabilities.

Although it would also be possible to combine the copulas shown in Table 1 in the four-dimensional construction, we will concentrate on the same copula for all three generating functions in the construction scheme (Equation (12)) in order to analyze the pure effects of different types of tail dependence. Thus, the generators $\phi_{1}, \phi_{2}$, 


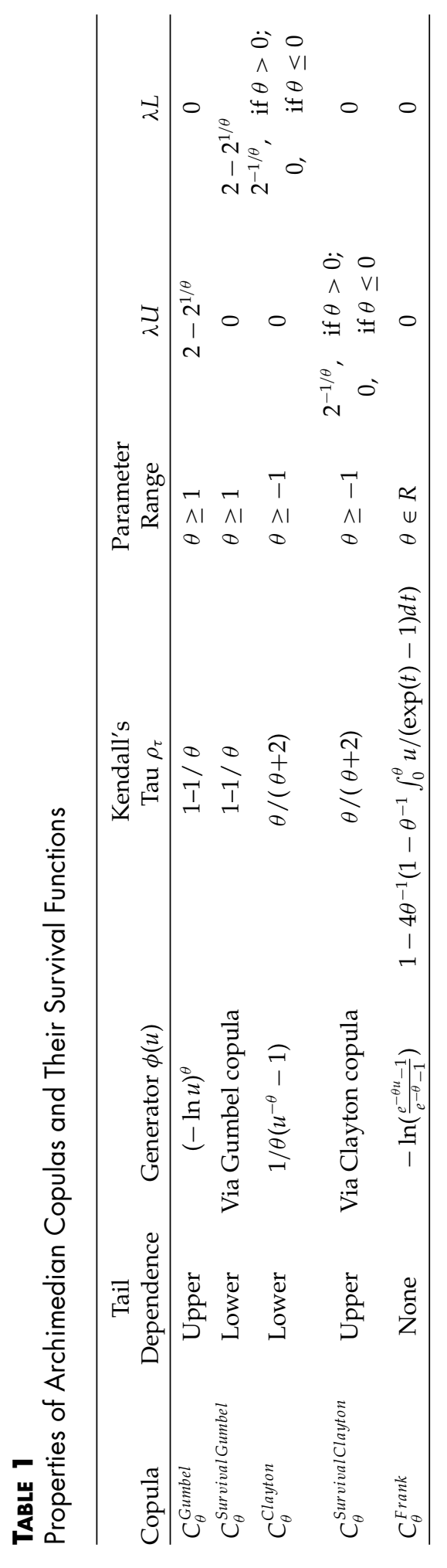




\section{Figure 1}

Dependence Structure of Nonexchangeable Archimedean Copulas

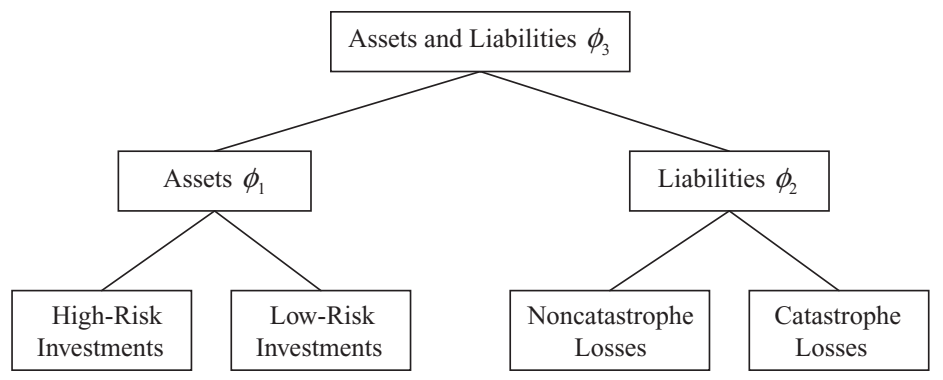

and $\phi_{3}$ differ only in their respective parameter values, which are calibrated using Kendall's rank correlation $\rho_{\tau}$.

We will use the generator function $\phi_{1}$ and its corresponding parameter $\theta_{1}$ to model the correlation between high-risk and low-risk investments, $\phi_{2}$ with parameter $\theta_{2}$ for the correlation between noncatastrophe losses and catastrophe losses, and $\phi_{3}$ with $\theta_{3}$ to correlate assets and liabilities. The copula parameter values $\theta_{1}, \theta_{2}$, and $\theta_{3}$ are calibrated based on the correlations $\rho_{\tau 1}$ (high- and low-risk investments), $\rho_{\tau 2}$ (noncatastrophe losses and catastrophe losses), and $\rho_{\tau 3}$ (assets and liabilities).

To generate random deviates from the Archimedean copulas, we apply the inverse transform method to the conditional distributions using numerical rootfinding techniques following the algorithm described in Embrechts, Lindskog, and McNeil (2001)..$^{5}$ An application of this algorithm to a financial market context can be found in Savu and Trede (2006). Berg and Aas (2007) compare the nonexchangeable Archimedean model with a pair-copula construction, examine estimation as well as simulation techniques and test the goodness-of-fit with two data sets. Nonexchangeable Archimedean copulas are computationally demanding and usually result in clumsy expressions. Therefore, we restrict ourselves to the basic description in Table 1. Nonexchangeable Archimedean copulas following the construction scheme of Equation (12) result in a hierarchical dependence structure that can be represented by a tree diagram, as shown by Figure 1.

One of the technical requirements in the construction of nonexchangeable Archimedean copulas results in higher correlations for copulas on a lower level in the hierarchical structure. This technical condition limits the level of correlation at higher hierarchical levels (see Joe, 1997, pp. 89-91). In our model the correlation between assets and liabilities must thus be smaller than the minimum of correlations of different asset classes and the correlations of different liability classes. Note that we concentrate on spatial correlation at certain points in time and do not model an intertemporal correlation on aggregate risk level. The only intertemporal correlation is at the premium level via the underwriting cycle.

\footnotetext{
${ }^{5}$ An alternative algorithm using Laplace transforms is presented in McNeil (2007). We also implemented this modeling approach. See Berg and Aas (2007) for a comparison of the different approaches.
} 


\section{Figure 2}

Lower Tail Dependence for the Survival Gumbel, the Clayton Copula, and the $t$ Copula

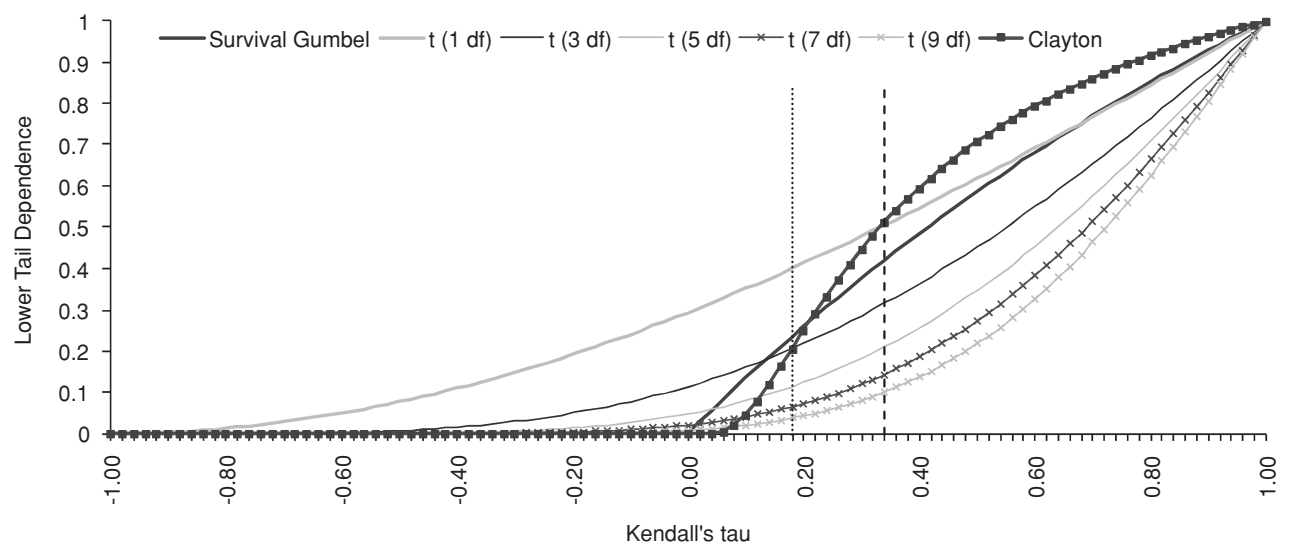

In the simulation analysis, the tail dependent copulas are each calibrated to the same set of Kendall's tau values so as to make them comparable. An advantage of using Kendall's tau for calibration is that it is more readily available than other measures of dependence, such as, e.g., the tail dependence coefficient $\lambda$. For the Gumbel and Clayton copulas, tail dependence can be fully determined by Kendall's tau (see Table 1 , columns 5 and 6), but for the $t$ copula, tail dependence involves both Kendall's tau and the degrees of freedom $v$ chosen. ${ }^{6}$ The higher Kendall's tau, the higher the tail dependence. Moreover, the fewer the degrees of freedom in the $t$ copula, the higher the tail dependence. For degrees of freedom $\rightarrow \infty$, the $t$ copula converges to the Gaussian copula and the tail dependence converges toward 0.

Focusing on lower tail dependence, Figure 2 compares the Survival Gumbel, the Clayton, and the $t$ copulas for different degrees of freedom (1 to 9) for a bivariate couple in the four-variate nonexchangeable copula. ${ }^{7}$ The figure also illustrates the range of lower tail dependence that can be achieved with the different copulas. For the Clayton, the Survival Gumbel, and the $t$ copulas the range goes from 0 to 1 ; for a negative Kendall's tau, the lower tail dependence of the Clayton and the Survival Gumbel copula is 0 . Upper tail dependence is similar to the dependence shown in Figure 2, with Survival Gumbel replaced by Gumbel and Clayton replaced by Survival Clayton.

The degrees of freedom can be chosen so that the lower tail dependence of the $t$ copula matches the lower tail dependence of the Clayton copula or of the Survival Gumbel. For example, if Kendall's tau is 0.18 (dotted line in Figure 2), the lower tail dependence of the $t$ copula with three degrees of freedom corresponds to the

\footnotetext{
${ }^{6}$ The $t$ copula is symmetric and its tail dependence is given by $2 t_{v+1}(-\sqrt{(v+1)(1-\rho) /(1+\rho)})$; see Equation (11) for the relationship between $\rho$ and Kendall's tau. Note that the Gauss and Frank copulas do not exhibit tail dependence and therefore are not mentioned in this context. ${ }^{7}$ In the later simulations (the "Measuring the Influence of Copulas in DFA" section) the Survival function for one of the variates is used in order to obtain negative dependence for the Survival Gumbel copula.
} 
lower tail dependence of the Clayton copula. If Kendall's tau is 0.34 (dashed line in Figure 2), the $t$ copula with one degree of freedom would lead to the same lower tail dependence as the Clayton copula.

In our simulation analysis we present results for five different degrees of freedom $(1,3,5,7$, and 9). We do this because in our model we vary Kendall's tau for the different assets, liabilities, and between assets and liabilities and we thus need different degrees of freedom to match the lower tail dependence of the $t$ and Clayton copulas. However, since we generate random numbers from one multidimensional $t$ distribution, we can choose only one degree of freedom parameter for the whole model. Therefore, we can calibrate the $t$ copula to exactly match the Clayton copula only for the special case when Kendall's tau is identical for all three dependencies being considered. ${ }^{8}$ We use two different approaches to deal with this technical problem. First, we show the full spectrum of possible outcomes by considering different degrees of freedom (see the "Measuring the Influence of Copulas in DFA" section). Second, we present two examples where the lower tail dependence of the $t$ and Clayton copulas corresponds to each other (see the Appendix, where we consider an identical Kendall's tau of $0.1(0.2)$ and 7.00 (2.83) degrees of freedom, which leads to a lower tail dependence of 0.04 (0.25) for both the $t$ and Clayton copulas).

\section{Measurement of Risk, Return, and Performance in DFA}

\section{Return}

In the simulation study, we measure risk, return, and performance considering seven financial ratios used in Eling, Parnitzke, and Schmeiser (2008). As a measure of return, we consider the expected gain per annum. We denote the expected gain from time 0 to time $T$ as $E\left(E C_{T}\right)-E C_{0}$. The expected gain $E(G)$ per annum can be written as

$$
E(G)=\frac{E\left(E C_{T}\right)-E C_{0}}{T}
$$

\section{Risk}

We analyze three risk measures: standard deviation, ruin probability, and expected policyholder deficit (results for value at risk and tail value at risk (see Dowd and Blake, 2006) are available upon request). The standard deviation of the gain per annum $\sigma(G)$ takes into account both positive and negative deviations from the expected value and

\footnotetext{
${ }^{8}$ Due to the hierarchical structure of the problem, we cannot calculate tail dependence for the full model, as we use pairwise tail dependence on the different hierarchical levels. We also cannot apply the $t$ copula with multiple parameters of degrees of freedom as presented, e.g., in Luo and Shevchenko (2007), because this approach can be used only to calibrate within groups (Kendall's tau for the different assets or for the different liabilities) but not between groups (Kendall's tau between assets and liabilities; for the grouped $t$ copula; see also McNeil, Frey, and Embrechts, 2005). Schmid and Schmidt (2007) consider multivariate extensions of Spearman's rho and of tail dependence measures, which could be used to extend our model when there are more than two elements in one hierarchical group (e.g., more than two asset classes or more than two groups of liabilities).
} 
thus is a measure of total risk:

$$
\sigma(G)=\frac{\sigma\left(E C_{T}\right)}{T}
$$

However, in the insurance context, risk is often measured using downside risk measures such as the ruin probability $(R P)$ or the expected policyholder deficit $(E P D)$. Downside risk measures differ from total risk measures in that only negative deviations from a certain threshold are taken into account. In this context, the ruin probability can be defined as

$$
R P=\operatorname{Pr}(\hat{\tau} \leq T),
$$

where $\hat{\tau}=\inf \left\{t>0 ; E C_{t}<0\right\}$ with $t=1,2, \ldots, T$ describes the first occurrence of ruin (i.e., a negative equity capital; see, e.g., Heilmann, 1988, p. 247). Note that the calculation of ruin probabilities in discrete time leads to lower values than does continuous-time analysis (see, e.g., Bühlmann, 1996, p. 134). However, closed-form solutions of ruin problems are generally available only under very restrictive conditions (see Rolski et al., 1998, p. 19). Thus, for more complex models, analytical or numerical approximations are necessary. An argument in favor of simulation models in discrete time is that they make it easy to consider dependencies of differently distributed risks (see, e.g., McNeil, Frey, and Embrechts, 2005, p. 232).

The ruin probability does not provide any information regarding the severity of insolvency (see Butsic, 1994; Barth, 2000) or the time value of money (see Powers, 1995; Gerber and Shiu, 1998). To take these into account, the EPD can be considered:

$$
E P D=\sum_{t=1}^{T} E\left[\max \left(-E C_{t}, 0\right)\right]\left(1+r_{f}\right)^{-t}
$$

where $r_{f}$ stands for the risk-free rate of return.

\section{Performance}

We consider three performance measures. The Sharpe ratio $S R_{\sigma}$ measures the relationship between the risk premium (mean excess return above the risk-free interest rate) and the standard deviation of returns (see Sharpe, 1966):

$$
S R_{\sigma}=\frac{E\left(E C_{T}\right)-E C_{0} \cdot\left(1+r_{f}\right)^{T}}{\sigma\left(E C_{T}\right)} .
$$

In the numerator, the risk-free return is subtracted from the expected value of the equity capital in $T$. Using the standard deviation as a measure of risk, the Sharpe ratio also measures positive deviations of the returns in relation to the expected value. Since risk is often calculated by downside measures, either the ruin probability or the EPD can be used in the denominator of the Sharpe ratio: 


$$
\begin{gathered}
S R_{R P}=\frac{E\left(E C_{T}\right)-E C_{0} \cdot\left(1+r_{f}\right)^{T}}{R P}, \\
S R_{E P D}=\frac{E\left(E C_{T}\right)-E C_{0} \cdot\left(1+r_{f}\right)^{T}}{E P D} .
\end{gathered}
$$

$S R_{R P}$ denotes the Sharpe ratio based on ruin probability. $S R_{E P D}$ is the Sharpe ratio based on expected policyholder deficit.

\section{Measuring the Influence of Copulas in DFA}

\section{Model Specifications}

In our simulation study we present results for a stylized example so as to focus directly on the methodology. An application of our methodology to real-world data is available upon request (the "real-world" application uses data from a German nonlife insurance company). Table 2 summarizes the model parameters, their definitions, and their initial values for the stylized example.

In the simulation study we consider a time horizon of 5 years. The market volume $M V_{t}$ of the underwriting market accessible to the insurance company is $€ 1,000$ million and rises by $i=3$ percent each year. Assets are normally distributed, noncatastrophe losses are log-normally distributed, and the catastrophe claims are modeled using a Pareto distribution. ${ }^{9}$ The underwriting cycle is parameterized using the German alllines underwriting profit ratios as an example. ${ }^{10}$ The consumer response parameter $c r$ is $1(0.95)$ if the equity capital at the end of the last period is above (below) the company's safety level. The company's safety level is determined by the minimum capital required $(M C R)$, which is based on the Solvency I rules in effect in Germany.

Another important set of input parameters in our simulation are the correlation assumptions. There is no clear empirical evidence concerning these correlation values (see Lambert and Hofflander, 1966; Haugen, 1971; Kahane and Nye, 1975; Li and Huang, 1996); however, in the robustness tests we will present results for alternative

\footnotetext{
${ }^{9}$ To avoid the situation where the Pareto distribution generates infinitely large losses, we define a maximum loss based on the concept of the probable maximum loss (see Woo, 2002). Although the ruin probability is hardly affected by the choice of this cutoff point, the level of EPD depends on the maximum loss. However, additional robustness tests show that the relationship between the different copulas is not affected by the choice of the maximum loss. In additional tests, we also varied the distributional assumptions and, e.g., considered the normal inverse Gaussian and skewed $t$ distribution for the assets, the Gamma for the noncatastrophe losses, and the Frechet for the catastrophe losses. See Bali and Theodossiou (2008) for the risk measurement performance of alternative distribution functions.

${ }^{10}$ The data are taken from Cummins and Outreville (1987). A cycle will be present if $a_{1}>0$, $a_{2}<0$, and $a_{1}^{2}+4 a_{2}<0$. These statonarity conditions imply that underwriting profits follow a cyclical pattern. The cycle period can then be obtained by $2 \pi / \cos ^{-1}\left(a_{1} / 2 \sqrt{-a_{2}}\right)$. In our case, the cycle period equals 7.76 years. This cycle will either have a tendency to die down over time if $\sqrt{-a_{2}}<1$ or be explosive if $\sqrt{-a_{2}}>1$. Even a damped cycle will be maintained over time if random shocks occur.
} 


\section{TABLE 2}

\section{Parameter Configuration for Stylized Example}

\begin{tabular}{|c|c|c|}
\hline Parameter & Symbol & $\begin{array}{l}\text { Initial Value } \\
\text { at } t=0\end{array}$ \\
\hline Time period in years & $T$ & 5 \\
\hline Equity capital at the end of period $t$ & $E C_{t}$ & $€ 75$ million \\
\hline Tax rate & $\operatorname{tr}$ & 0.25 \\
\hline Portion invested in high-risk investments in period $t$ & $\alpha_{t-1}$ & 0.40 \\
\hline Normally distributed high-risk investment return in period $t$ & $r_{1 t}$ & \\
\hline Mean return & $E\left(r_{1 t}\right)$ & 0.10 \\
\hline Standard deviation of return & $\sigma\left(r_{1 t}\right)$ & 0.20 \\
\hline Normally distributed low-risk investment return in period $t$ & $r_{2 t}$ & \\
\hline Mean return & $E\left(r_{2 t}\right)$ & 0.05 \\
\hline Standard deviation of return & $\sigma\left(r_{2 t}\right)$ & 0.05 \\
\hline Risk-free return & $r_{f}$ & 0.03 \\
\hline Underwriting market volume & $M V$ & $€ 1,000$ million \\
\hline Market growth & $i$ & 0.03 \\
\hline Company's underwriting market share in period $t$ & $\beta_{t-1}$ & 0.20 \\
\hline Premium rate level in period $t$ & $\Pi_{t}$ & 1 \\
\hline Autoregressive process parameter for lag 0 & $a_{0}$ & 1.191 \\
\hline Autoregressive process parameter for lag 1 & $a_{1}$ & 0.879 \\
\hline Autoregressive process parameter for lag 2 & $a_{2}$ & -0.406 \\
\hline Consumer response function & $\operatorname{cr}_{t-1}^{E C_{t-1}}$ & 1 \\
\hline $\begin{array}{l}\text { Upfront expenses linearly depending on the written market } \\
\text { volume }\end{array}$ & $\stackrel{t-1}{\gamma}$ & 0.05 \\
\hline $\begin{array}{l}\text { Upfront expenses nonlinearly depending on the change in } \\
\text { written market volume }\end{array}$ & $\eta$ & 0.001 \\
\hline $\begin{array}{l}\text { Log-normal noncatastrophe claims as portion underwriting } \\
\text { market share }\end{array}$ & $C_{\text {ncat }}$ & \\
\hline Mean claims & $E\left(C_{n c a t}\right)$ & $€ 170$ million \\
\hline Standard deviation of claims & $\sigma\left(C_{n c a t}\right)$ & $€ 17$ million \\
\hline Claim settlement costs as portion of claims & $\delta$ & 0.05 \\
\hline Pareto distributed catastrophe claims & $C_{c a t}$ & \\
\hline Mean claims & $E\left(C_{c a t}\right)$ & $€ 0.5$ million \\
\hline Dispersion parameter & $D\left(C_{c a t}\right)$ & 4.5 \\
\hline $\begin{array}{l}\text { Kendall's rank correlation between high- and low-risk } \\
\text { investments }\end{array}$ & $\rho_{\tau 1}$ & 0.2 \\
\hline $\begin{array}{l}\text { Kendall's rank correlation between noncatastrophe losses } \\
\text { and catastrophe losses }\end{array}$ & $\rho_{\tau 2}$ & 0.2 \\
\hline Kendall's rank correlation between assets and liabilities & $\rho_{\tau 3}$ & -0.1 \\
\hline
\end{tabular}

parameter settings. In our stylized example, we use random numbers with the following correlation structure. Kendall's rank correlation between high- and low-risk investments is 0.2 , the correlation between catastrophe losses and noncatastrophe losses is 0.2 , and between assets and liabilities Kendall's rank correlation is $-0.1 .^{11}$

\footnotetext{
${ }^{11}$ To generate the negative dependence between assets and liabilities, we apply the uniform random variates generated with the hierarchical Archimedean copulas to the Survival functions of the marginal distributions for the liabilities.
} 


\section{Simulation Results}

Table 3 sets out the simulation results for six different dependence structures. All results have been calculated on basis of a Monte Carlo simulation with 500,000 iterations (for details on Monte Carlo simulation, see, e.g., Glassermann, 2004).

In the case without correlations (i.e., we assume independence between assets, liabilites, and assets and liabilities), we find an expected gain of $€ 31.51$ million per annum with a standard deviation of $€ 13.57$ million. The ruin probability amounts to 0.09 percent. This corresponds to an investment grade rating and is below the requirements of many regulatory authorities (e.g., the Solvency II framework planned in the European Union requires a ruin probability below 0.50 percent; see European Commission, 2008).

Comparing the case without correlations and the Gauss copula (i.e., only linear dependence is considered), we observe minor effects on the mean returns. $E(G)$ is reduced about 1.24 percent, from $€ 31.51$ million to $€ 31.12$ million. However, we find much larger changes in risk. $\sigma(G)$ rises from $€ 13.57$ million to $€ 16.04$ million $(+18.20$ percent) and $R P$ from 0.09 percent to 0.42 percent ( +466.67 percent). Obviously, the extreme changes in risk are especially due to the lower partial moments, as the increase in ruin probability (the measure for downside risk) is 26 times higher than the increase in standard deviation (the measure for total risk). Therefore, the performance is much lower than in the case without correlations: $S R_{\sigma}$ is reduced about 16.52 percent and $S R_{R P}$ by about 78.15 percent.

We find large differences when comparing the results for the copulas. Looking at the $t$, the Gumbel, the Clayton, and the Frank copulas, we again observe only minor effects on the mean returns and extreme effects on the risk. However, the change in risk depends on the form of nonlinear dependence. With the copulas that exhibit upper or no tail dependence (the Gumbel and Frank copulas), the ruin probability is lower than with the Gauss copula, whereas these values are much higher with lower tail dependent copulas (the $t$ and Clayton copulas). These findings are confirmed by the results of the Survival copulas. The Frank copula, which is the only symmetric Archimedean copula, exhibits a lower ruin probability than the Gauss copula as it is lighter in the tails (see Venter, 2002). It is also noteworthy that in this example the ruin probability for the $t$, the Survival Gumbel, and for the Clayton copulas are above most regulatory requirements (e.g., 0.50 percent in Solvency II).

The impact of nonlinear dependencies on different risk measures can be illustrated by the results for ruin probability and expected policyholder deficit. The Gumbel copula has a smaller $R P$ compared to the Gauss and the $t$ copulas, but the EPD is much higher; comparing the performance measures based on downside risk, we find that the Gumbel copula has a higher $S R_{R P}$ than the Gauss and the $t$ copulas, but a lower $S R_{E P D}$. These differences might be due to the extreme levels of the higher moments (skewness, kurtosis) with the nonsymmetric Gumbel copula, as the EPD is more sensitive to higher moments than is the ruin probability. ${ }^{12}$ The results illustrate the

\footnotetext{
${ }^{12}$ The EPD is much higher with the nonsymmetric copulas compared to the symmetric Gauss, $t$, and Frank copulas because nonsymmetric copulas generate more extreme values in the tails compared to their symmetric counterparts. For example, based on lower tail dependence,
} 


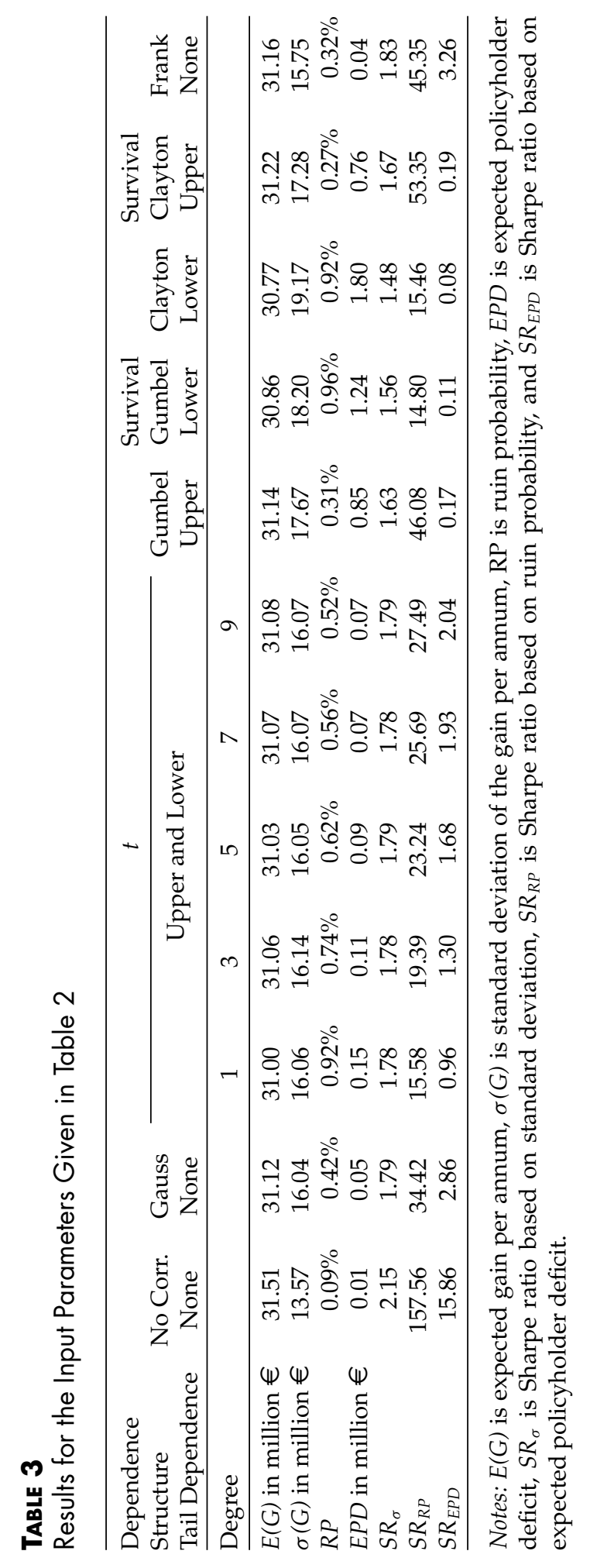


importance of modeling nonlinear dependencies in DFA, as the integration of these features has extreme effects on the risk and performance of the insurance company. The results found with the $R P$ and the EPD might be of special relevance for regulators and rating agencies because, depending on the copula concept employed, we find large differences in risk assessment for different risk measures.

\section{Robustness of Findings}

We check the robustness of our findings by varying the level of equity capital, the correlation settings, and the time horizon. The results are robust if the basic relations between the analyzed copulas are independent of the given input parameters.

In the first step, we vary the level of equity capital in $t=0$, which determines the company's safety level, leaving everything else constant. In the "Simulation Results" section, the level of equity capital was set at $€ 75$ million. To test the implications of different levels of equity capital, we vary the equity capital in $t=0$ from $€ 50$ to $€ 100$ million in $€ 5$ million intervals. The results are shown in Figure 3, where the ruin probability and the expected policyholder deficit are displayed as a function of the equity capital.

As the level of equity capital increases, the ruin probability decreases with all copulas because the company's safety level is improved. However, the relative difference between the copulas increases with an increasing level of equity capital. For example, with $E C_{0}=50$, the ruin probability of the Clayton copula ( $R P=2.62$ percent $)$ is five times higher than the ruin probability without correlation $(R P=0.49$ percent), but with $E C_{0}=100$, the ruin probability of the Clayton copula $(R P=0.316$ percent $)$ is 26 times higher than in the case without correlation $(R P=0.012$ percent). The fact that the influence of nonlinear dependencies increases with an increasing level of equity capital is an important result because it indicates that copulas are relevant not only for low-capitalized companies but also for well-capitalized companies.

Looking at the expected policyholder deficit, we find a relatively small risk reduction especially with the Gumbel and the Clayton copulas. As mentioned, the asymmetric Gumbel and Clayton copulas generate more extreme values compared to the symmetric copulas and these high values are not affected by an increase in equity capital; thus, the EPD is hardly affected by a change in equity capital. Considering the EPD, the results of the simulation thus indicate that the risks generated by tail dependencies are not much reduced by an increasing level of equity capital. Therefore, it again seems that copulas are important for well-capitalized companies. This result is also relevant for policyholders and regulators because the expected policyholder deficit is more important for the policyholders than for the equity holders (see Bingham, 2000), given that policyholders have to bear the amount of loss, whereas the shareholders (in case of limited liability) have a limited downside risk.

we would expect that the risk of the $t$ copula with three degrees of freedom is comparable to the risk of the Clayton copula. However, this is only true for the ruin probability but not for the EPD. Therefore, the extent of lower tail dependence corresponds to the ruin probability, but not to the $E P D$, which is much more sensitive to the extreme values produced by the nonsymmetric Gumbel and Clayton copulas. The EPD reflects both the lower tail dependence and the asymmetry of the copulas. 


\section{Figure 3}

Variation of Equity Capital in $t=0$ Between $€ 50$ and $€ 100 \mathrm{~m}(R P$ and $E P D)$

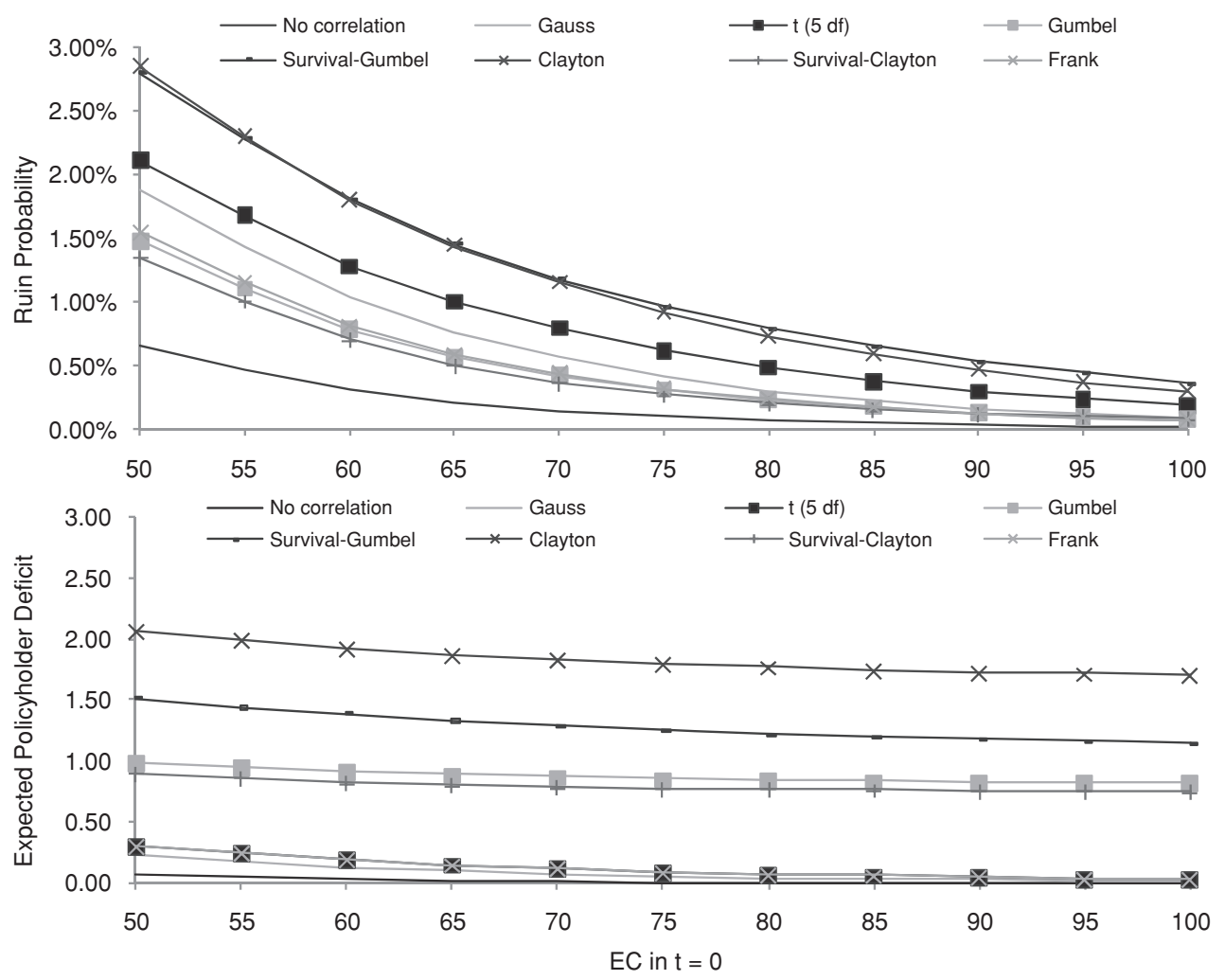

In the second step, we vary the correlations. In our basic setting, these are relatively low. Kendall's rank correlation between high- and low-risk investments is 0.2 , between catastrophe losses and noncatastrophe losses 0.2 , and between assets and liabilities -0.1 . To test the implications of different correlation assumptions on the level of ruin probability, we vary the correlation between the high- and low-risk investments from 0.1 to 0.5 in 0.1 intervals (upper part of Figure 4 ) and between catastrophe losses and noncatastrophe losses also from 0.1 to 0.5 in 0.1 intervals (lower part of Figure 4).

We find that the ruin probability increases with an increasing correlation between the assets. This occurs because the higher the correlation, the higher the likelihood that negative outcomes are generated for both types of assets (i.e., low returns with the high- and the low-risk investments). With the Clayton copula the increase is larger than with the Survival Gumbel because the relative increase in lower tail dependence is much higher (when Kendall's tau is increased; see Figure 2). All other relations remain robust. The increase in ruin probability is much smaller when the correlation between the liabilities is varied. This is because, with the given parametrization, the underwriting business is more profitable than the insurer's investments on the capital market. 


\section{Figure 4}

Variation of Correlation Between 0.1 and $0.5(R P)$
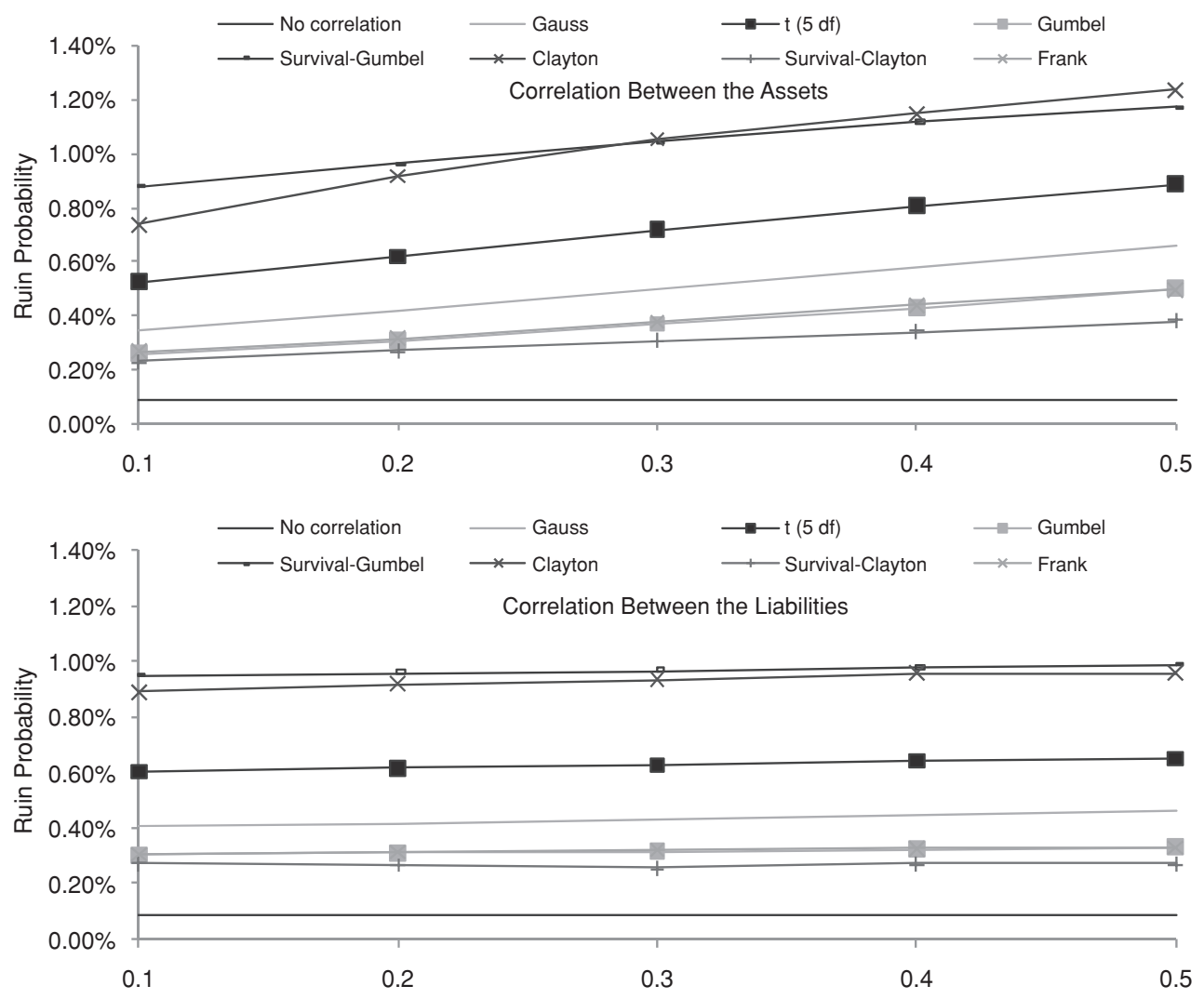

In the "Simulation Results" section, a time period of $T=5$ years was considered. To check how different time horizons affect our results, Figure 5 presents ruin probability and expected policyholder deficit for time horizons ranging from 1 to 20 years.

Both the ruin probability and the expected policyholder deficit increase when the time horizon is expanded. However, all the basic relations set out in the "Simulation Results" section between the different copulas remain unchanged; thus, the results are robust with regard to variation of the time horizon.

\section{Measuring the Influence of Risk Management Strategies}

\section{Model Specifications}

In this section, we investigate whether management can influence the risk introduced by tail dependencies. To this end, in our model, decisions concerning the portion of risky investments $(\alpha)$ and the market share in the underwriting business $(\beta)$ can be made at the beginning of each year. We first consider two management strategies introduced by Eling, Parnitzke, and Schmeiser (2008): the solvency strategy and the growth strategy. 


\section{FIGURE 5}

Variation of Time Horizon Between 1 and 20 Years

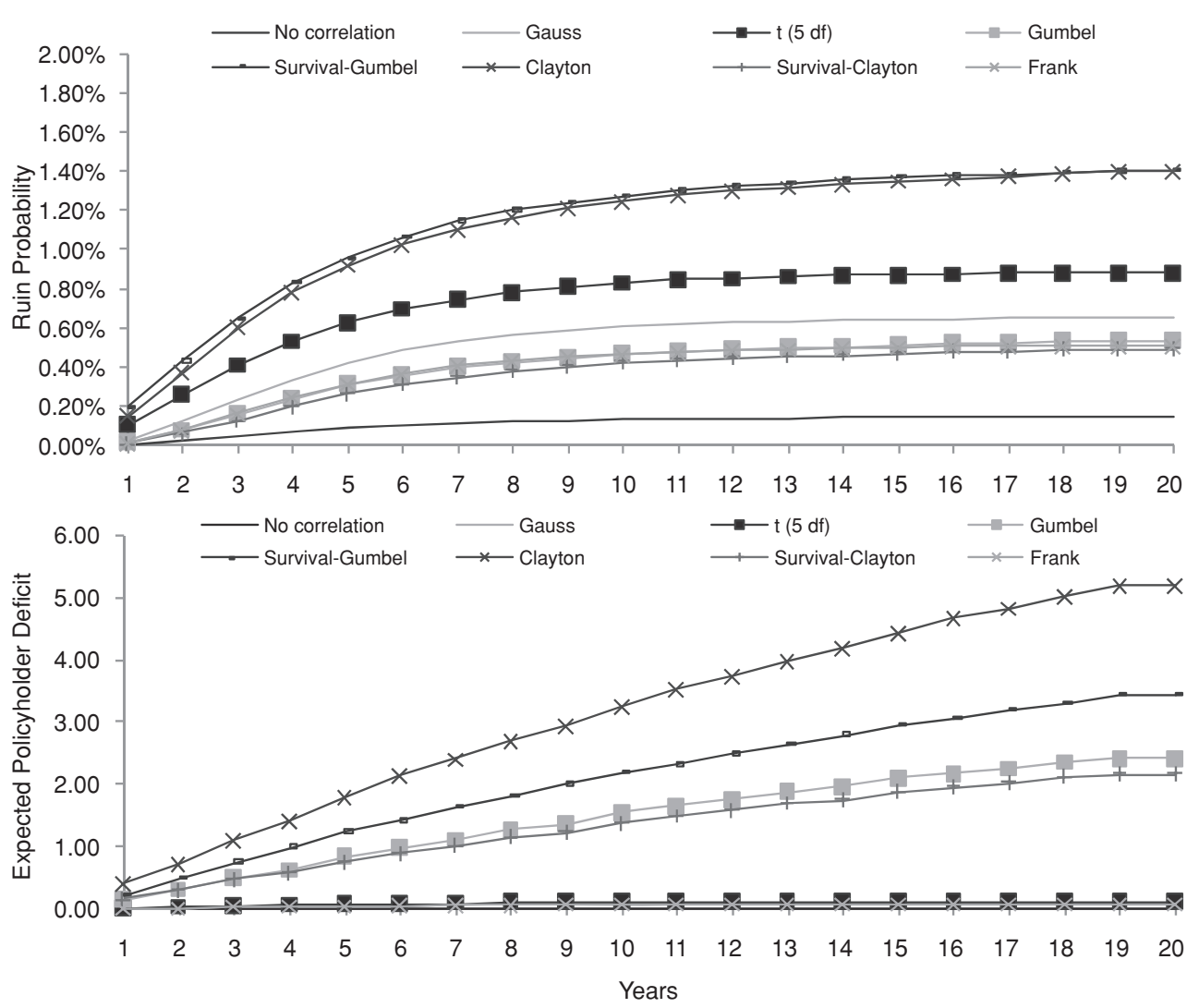

The solvency strategy is aimed at risk reduction. For each point in time $(t=1, \ldots, T-1)$, we decrease the portion of risky investments $\alpha$ by 0.05 and the insurance market share $\beta$ by 0.02 as soon as the equity capital falls below the critical value defined by the $M C R$ plus a safety loading of 50 percent. The growth strategy combines the solvency strategy with a growth target for the underwriting business. Should the equity capital drop below the $M C R$, including a safety loading of 50 percent, the same rules apply as in the solvency strategy. If the equity capital is above the trigger, there is a growth of 0.02 in $\beta$.

In addition to the strategies used in Eling, Parnitzke, and Schmeiser (2008), we consider a reinsurance strategy. Here the insurer signs a stop-loss reinsurance contract on its whole book of business with an attachment point of $€ 200$ million, a limit of $€ 40$ million, and a premium of $€ 4$ million at the beginning of each year. The payment from the reinsurer at the end of each year can thus be calculated by $\min \left(\max \left(C_{t}-200\right.\right.$, $0) ; 40)$.

Of course, these three strategies are only heuristic risk management approaches. We use them to provide some general insights into simple risk-reduction approaches under the different copulas. Further research on this topic might include analyzing 
different reinsurance contracts (such as stop loss, excess of loss, and double trigger contracts) and their effects on an insurers risk and return position (see Eling and Toplek, 2007). Another idea would be to search for an optimal risk management strategy within the DFA framework (see D'Arcy and Gorvett, 2004, for a related analysis).

\section{Simulation Results}

The simulation results for the solvency, growth, and reinsurance strategies are presented in Table 4 . When comparing the results of Table 4 with those of Table 3, we see that downside risk is reduced under the solvency strategy although the return remains almost unchanged. Thus, the solvency strategy reduces the ruin probability without having much effect on the return. However, risk is not as much reduced when nonlinear dependencies are taken into account. The solvency strategy is thus not effective in reducing downside risk in the case of nonlinear dependencies.

Under the growth strategy, we obtain a completely different risk and return profilea higher return is accompanied by higher risk. Again, the level of return is not affected by the integration of nonlinear dependencies, but large differences are found for downside risk measures. Here risk is much increased with all copula models. Therefore, the performance numbers for the growth strategy are mostly lower than those in the situation where no management rule is applied.

In contrast to the other strategies, the reinsurance strategy leads to a lower return. We again find large differences for the downside risk measures. The ruin probability is in all cases kept within the regulatory limits suggested by the Solvency II framework (the maximum ruin probability is 0.37 percent for the Survival Gumbel copula). It might thus seem that reinsurance is an efficient method to limit the risks generated by nonlinear dependencies. However, this is again only true from the equity holders' perspective, because we find that the EPD is very little reduced by purchasing reinsurance.

\section{Robustness of Findings}

We checked the robustness of our findings using the tests described in the previous "Robustness of Findings" section. Figure 6 shows the ruin probability for different levels of equity capital under the solvency strategy (upper part of the figure), the growth strategy (middle part of the figure), and the reinsurance strategy (lower part of the figure). The expected policyholder deficit yields the same conclusions as previously.

The results displayed in Figure 6 are comparable to the results presented in Figure 3 . As the equity capital increases, the ruin probability decreases for all strategies. The only difference is the base level of the ruin probability. The strategies cannot influence the relative difference between the copulas described in the "Simulation Results" section, i.e., the fact that the relative difference between the copulas increases with the level of equity capital. However, overall these results indicate that the main conclusions presented in the "Simulation Results" section are very robust. We also investigated the implications of different correlation assumptions and different time horizons and again found robust results. 


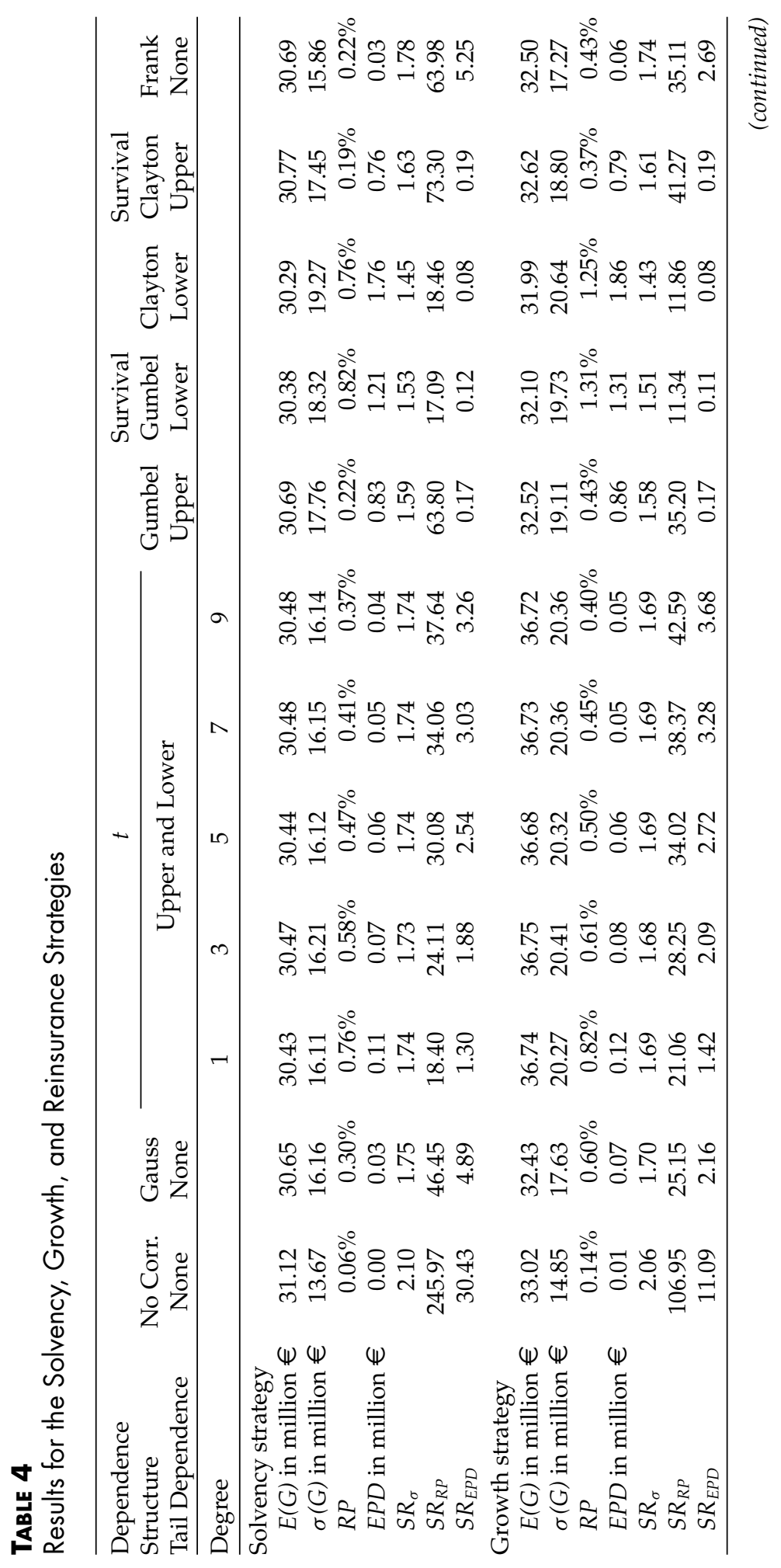




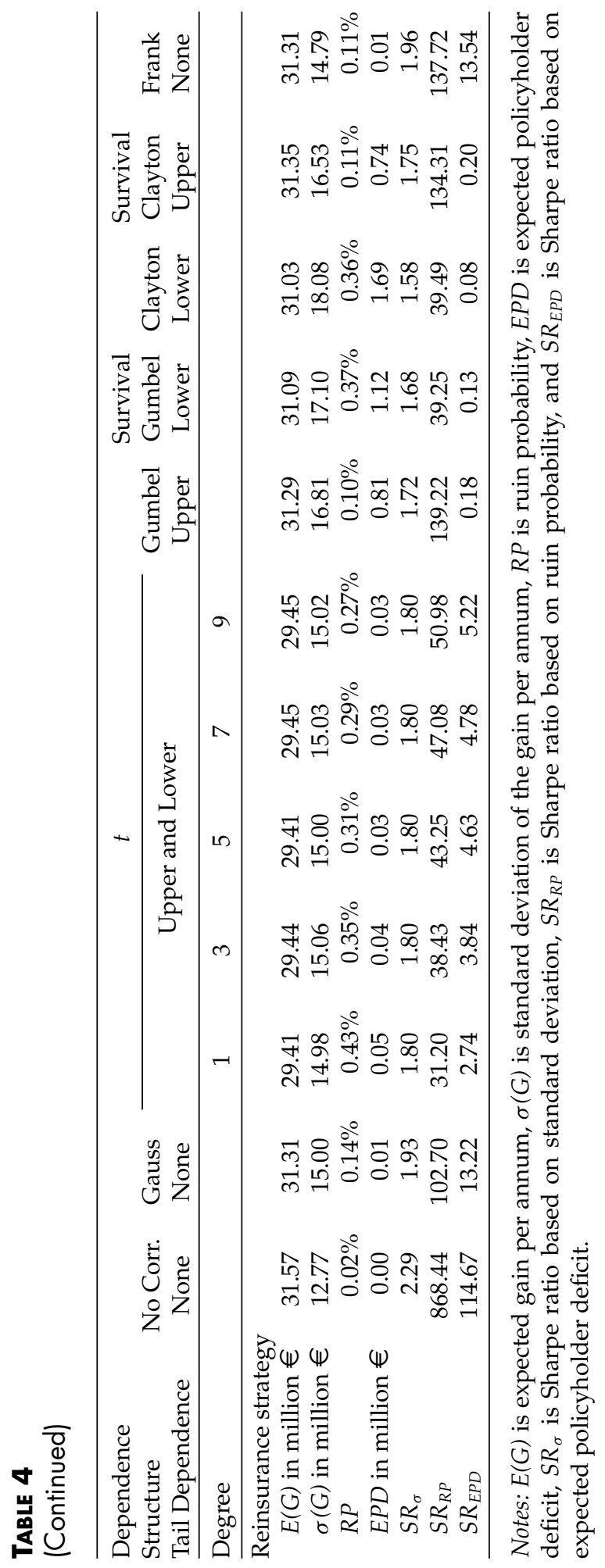




\section{Figure 6}

Variation of Equity Capital in $t=0$ Between $€ 50 \mathrm{~m}$ and $€ 100 \mathrm{~m}(R P)$
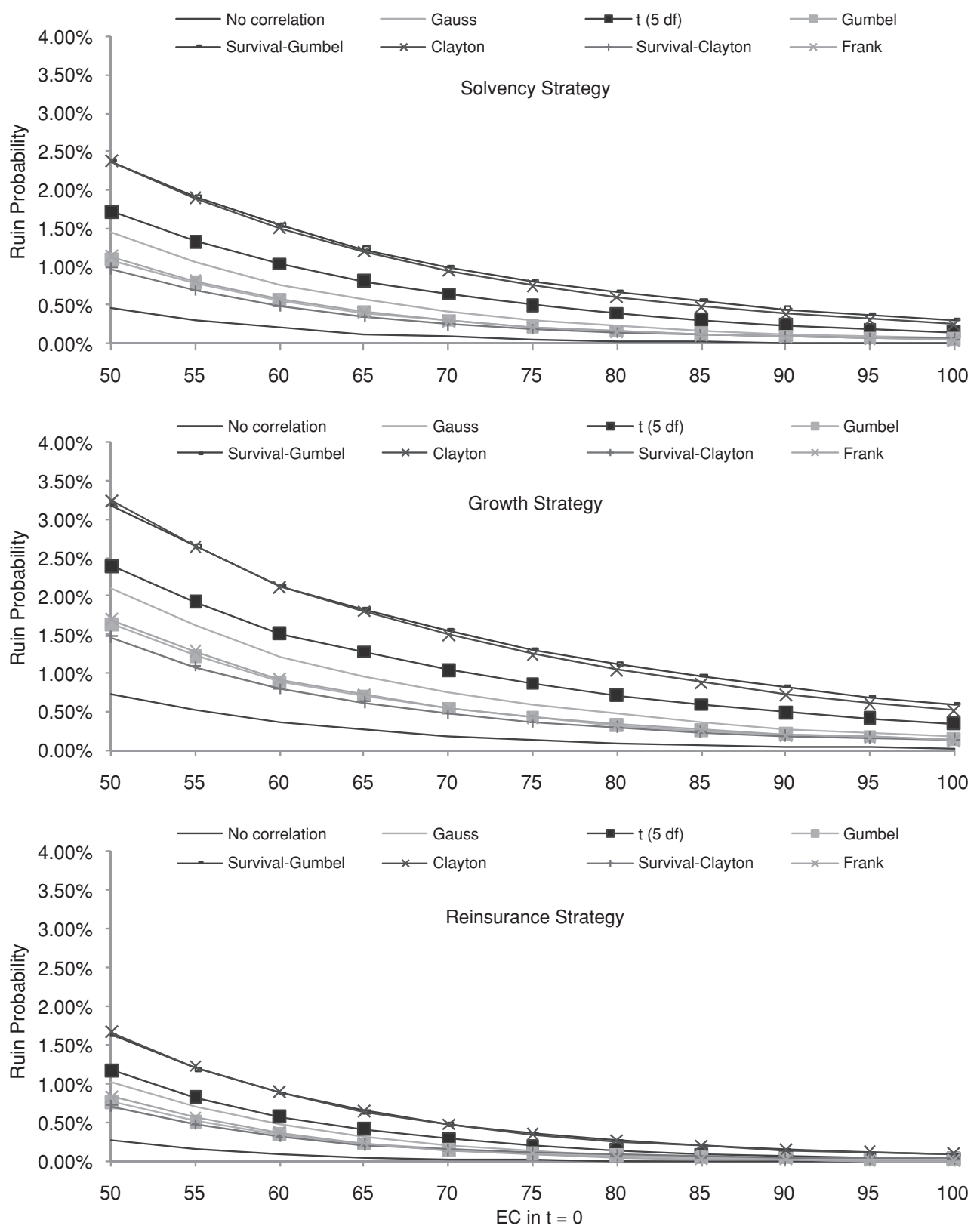

\section{CONCLUSION}

We study the influence of nonlinear dependencies on a non-life insurers risk and return profile by integrating several copula models in a DFA framework. Nonlinear dependencies are especially relevant in the case of extreme events that might induce tail dependencies between different assets, different kinds of liabilities, or between 
assets and liabilities. One example of such extreme events are the terrorist attacks of September 11, 2001, which resulted in insurers experiencing large losses from the underwriting business and on the capital markets.

We have three main findings, each with important implications for insurance company stakeholders. First, we find that extreme events are especially relevant for policyholders and regulators (which have to monitor insurers solvency to protect policyholders) because nonlinear dependencies do not affect the return level but, instead, the ruin probability and the expected policyholder deficit (EPD). Depending on the copula, the ruin probability increases up to a factor of 11 in our simulation study compared to a situation without dependencies. We observe the highest levels of risk in case of lower tail dependent copulas such as the $t$ and the Clayton copulas.

A second key result is that although in general the ruin probability decreases when equity capital increases, there are nonlinear dependencies where the expected policyholder deficit cannot be reduced by increasing equity capital-a finding that is again of special importance for policyholders and regulators. It thus seems that copulas are relevant not only for low-capitalized companies but also for well-capitalized companies. This finding also highlights the importance of considering nonlinear dependencies, especially for regulators and rating agencies. Depending on the copula concept employed, we find large differences in the risk assessment of policyholder deficit and ruin probability. As these measures are the foundation of capital standards and ratings, it is important to consider nonlinear dependencies in the regulatory framework and in rating assessment, e.g., in stress testing and scenario analysis. The ruin probability and EPD as well as related quantile based measures (value at risk, tail value at risk; see Dowd and Blake, 2006) are also the most common risk measures in internal risk models, emphasizing the relevance of the results for risk management in insurance and reinsurance companies.

Third, we check the effectiveness of different risk management strategies used in response to adverse outcomes generated by nonlinear dependencies, but we find that the risk profile cannot be affected by simple risk reduction strategies. A reinsurance strategy can delimit the ruin probability, but not the expected policyholder deficit. In our simulation study, the reinsurance strategy thus proves to be an useful instrument for securing the position of equity holders, but not necessarily for policyholders, which have to bear the amount of loss in case of insolvency.

The article points toward an important distinction between copula functions when it comes to modeling the ruin probability and the expected policyholder deficit, i.e., the distinction between symmetric and asymmetric dependence structures of different asset classes and different liabilities. Imposing a symmetric dependence structure leads to significantly lower EPD values for matched Kendall's tau and degrees of freedom parameters across copula specifications. The survival copulas confirm the higher sensitivity of the EPD to extreme tail events as compared to the ruin probability. These results emphasize the importance of modeling asymmetric nonlinear dependencies.

As mentioned, there are a number of empirical papers that attempt to answer the question of which copula will provide the best fit for empirical data that contain 
different asset classes and different liabilities. The general finding of all these studies is that it is not easy to fit parametric copulas to empirical data but that the Gaussian copula is most likely not the best one for describing real-world dependencies, thus giving our scenario analyses greater relevance. In practice, different business units of an insurance company have detailed data for their specific segment and are thus able to calculate a univariate loss distribution that is a reasonably accurate description of their business. These marginal models are then integrated into a multivariate model by assuming a correlation structure, which is typically Gaussian. This means that detailed marginal data are combined with rather scarce dependence information, an approach fraught with model risk. In this article, we provide an assessment of this risk by considering a specific selection of copulas.

Moreover, the current financial market crisis, which has impacted many insurers on both sides of their balance sheets, illustrates the importance of analyzing extreme events in an asset liability management context. It is also important that existing regulation in Europe (Solvency II, Swiss Solvency Test) incorporates linear dependencies but neglects nonlinear dependencies, which is also true of most internal risk models used in practice. Our article shows what can happen in extreme scenarios such as simultaneous adverse developments in different business areas, and we believe that the current crisis is a good real-world illustration of their relevance. As these scenarios are not fully reflected in current regulation and most of the internal models, we recommend their use in stress testing.

\section{APPENDIX}

Results for Matched Kendall's Tau and Degrees of Freedom

\begin{tabular}{|c|c|c|c|c|c|c|c|c|}
\hline \multirow{3}{*}{$\begin{array}{l}\text { Dependence } \\
\text { Structure } \\
\text { Tail Dependence }\end{array}$} & \multirow{3}{*}{$\begin{array}{l}\text { No Corr. } \\
\text { None }\end{array}$} & \multirow{3}{*}{$\begin{array}{l}\text { Gauss } \\
\text { None }\end{array}$} & \multicolumn{2}{|l|}{$t$} & \multicolumn{2}{|l|}{ Survival } & \multicolumn{2}{|l|}{ Survival } \\
\hline & & & Upper & Gumbel & Gumbel & Clayton & Clayton & Frank \\
\hline & & & and Lower & U Upper & Lower & Lower & Upper & None \\
\hline \multicolumn{9}{|c|}{ Kendall's tau $=0.1$ and degrees of freedom $=7.00$} \\
\hline$E(G)$ in million $€$ & 31.51 & 31.20 & 31.16 & 31.32 & 30.91 & 31.03 & 31.21 & 31.30 \\
\hline$\sigma(G)$ in million $€$ & 13.57 & 15.52 & 15.55 & 15.50 & 17.06 & 15.87 & 17.01 & 15.30 \\
\hline$R P$ & $0.09 \%$ & $0.33 \%$ & $0.46 \%$ & $0.26 \%$ & $0.85 \%$ & $0.73 \%$ & $0.25 \%$ & $0.25 \%$ \\
\hline$E P D$ in million $€$ & 0.01 & 0.04 & 0.06 & 1.28 & 1.04 & 1.64 & 0.87 & 0.04 \\
\hline$S R_{\sigma}$ & 2.15 & 1.86 & 1.85 & 1.87 & 1.67 & 1.80 & 1.69 & 1.89 \\
\hline$S R_{R P}$ & 157.56 & 43.45 & 31.43 & 56.16 & 16.76 & 19.76 & 58.67 & 57.58 \\
\hline$S R_{E P D}$ & 15.86 & 3.64 & 2.35 & 0.11 & 0.14 & 0.09 & 0.17 & 3.80 \\
\hline \multicolumn{9}{|c|}{ Kendall's tau $=0.2$ and degrees of freedom $=2.83$} \\
\hline$E(G)$ in million $€$ & 31.51 & 30.88 & 30.83 & 31.06 & 30.50 & 30.58 & 31.01 & 31.04 \\
\hline$\sigma(G)$ in million $€$ & 13.57 & 17.25 & 17.24 & 18.20 & 18.41 & 18.18 & 18.42 & 16.86 \\
\hline$R P$ & $0.09 \%$ & $0.80 \%$ & $1.08 \%$ & $0.50 \%$ & o $\quad 1.61 \%$ & $1.66 \%$ & $0.39 \%$ & $0.52 \%$ \\
\hline$E P D$ in million $€$ & 0.01 & 0.11 & 0.17 & 1.40 & 1.14 & 1.49 & 0.94 & 0.08 \\
\hline$S R_{\sigma}$ & 2.15 & 1.65 & 1.65 & 1.58 & 1.53 & 1.55 & 1.55 & 1.70 \\
\hline$S R_{R P}$ & 157.56 & 17.81 & 13.18 & 28.71 & 8.75 & 8.48 & 36.50 & 27.80 \\
\hline$S R_{E P D}$ & 15.86 & 1.35 & 0.86 & 0.10 & 0.12 & 0.09 & 0.15 & 1.81 \\
\hline
\end{tabular}




\section{References}

Achleitner, P. M., J. Biebel, and D. Wichels, 2002, Does WTC Matter for the Investment Policy of P/C Insurance Companies? Geneva Papers on Risk $\mathcal{E}$ Insurance-Issues $\mathcal{E}$ Practice, 27(2): 275-282.

Bali, T. G., and P. Theodossiou, 2008, Risk Measurement Performance of Alternative Distribution Functions, Journal of Risk and Insurance, 75(2): 411-437.

Barth, M. M., 2000, A Comparison of Risk-Based Capital Standards Under the Expected Policyholder Deficit and the Probability of Ruin Approaches, Journal of Risk and Insurance, 67(3): 397-414.

Berg, D., and K. Aas, 2007, Models for Construction of Multivariate Dependence, Working Paper, Norwegian Computing Center.

Bingham, R. E., 2000, Risk and Return: Underwriting, Investment, and Leverage, Proceedings of the Casualty Actuarial Society, 87(166): 31-78.

Blum, P., M. Dacorogna, P. Embrechts, T. Neghaiwi, and H. Niggli, 2001, Using DFA for Modeling the Impact of Foreign Exchange Risks on Reinsurance Decisions, Casualty Actuarial Society Forum, Summer: 49-93.

Blum, P., A. Dias, and P. Embrechts, 2002, The ART of Dependence Modeling: The Latest Advances in Correlation Analysis, in: M. Lane, ed., Alternative Risk Strategies (London: Risk Books).

Bühlmann, H., 1996, Mathematical Methods in Risk Theory, 2nd edition (Berlin: Springer).

Butsic, R. P., 1994, Solvency Measurement for Property-Liability Risk-Based Capital Applications, Journal of Risk and Insurance, 61(4): 656-690.

Casualty Actuarial Society, 2006, Dynamic Risk Modeling Handbook, CAS Dynamic Risk Modeling Working Party.

Charpentier, A., 2006, Dependence Structures and Limiting Results, With Applications in Finance and Insurance, Ph.D. thesis, Leuven.

Chen, R., K. A. Wong, and H. C. Lee, 1999, Underwriting Cycles in Asia, Journal of Risk and Insurance, 66(1): 29-47.

Chen, X., and Y. Fan, 2006, Estimation and Model Selection of Semiparametric CopulaBased Multivariate Dynamic Models Under Copula Misspecification, Journal of Econometrics, 135, 125-154.

Coles, S., J. Heffernan, and J. Tawn, 1999, Dependence Measures for Extreme Value Analysis, Extremes 2(4): 339-365.

Cummins, J. D., and J. F. Outreville, 1987, An International Analysis of Underwriting Cycles in Property-Liability Insurance, Journal of Risk and Insurance, 54(2): 246262.

D'Arcy, S. P., and R. Gorvett, 2004, The Use of Dynamic Financial Analysis to Determine Whether an Optimal Growth Rate Exists for a Property-Liability Insurer, Journal of Risk and Insurance, 71(4): 583-615.

D'Arcy, S. P., R. W. Gorvett, T. E. Hettinger, and R. J. Walling, 1998, Using the Public Access Dynamic Financial Analysis Model: A Case Study, CAS Dynamic Financial Analysis Call Paper Program, Summer: 53-118. 
Daykin, C. D., T. Pentikäinen, and M. Pesonen, 1994, Practical Risk Theory for Actuaries (London: Chapman \& Hall).

Demarta, S., and A. J. McNeil, 2005, The $t$ Copula and Related Copulas, International Statistical Review, 73(1): 111-129.

Dias, A., 2004, Copula Inference for Finance and Insurance. Doctoral Thesis ETH No. 15283, Zurich.

Dowd, K., and D. Blake, 2006, After VaR: The Theory, Estimation, and Insurance Applications of Quantile Based Risk Measures, Journal of Risk and Insurance, 73(2): 193-229.

Eling, M., and D. Toplek, 2007, Risk and Return of Reinsurance Under Copula Models, Working Paper, University of St. Gallen.

Eling, M., T. Parnitzke, and H. Schmeiser, 2008, Management Strategies and Dynamic Financial Analysis, Variance, 2(1): 52-70.

Embrechts, P., 2009, Copulas: A Personal View, Journal of Risk and Insurance, 76(3): 639-650.

Embrechts, P., P. Lindskog, and A. McNeil, 2001, Modeling Dependence With Copulas and Applications to Risk Management, in: S. T. Rachev, ed., Handbook of Heavy Tailed Distributions in Finance (Amsterdam: Elsevier), 329-384.

Embrechts, P., A. McNeil, and D. Straumann, 2002, Correlation and Dependence in Risk Management: Properties and Pitfalls, in: M. A. H. Dempster, ed., Risk Management: Value at Risk and Beyond (Cambridge, UK: Cambridge University Press), 176-223.

European Commission, 2008, Directive of the European Parliament and the Council on the Taking-Up and Pursuit of the Business of Insurance and Reinsurance-Solvency II, COM(2008) 119 final, Brussels.

Frees, E. W., and E. A. Valdez, 1998, Understanding Relationships Using Copulas, North American Actuarial Journal, 2(1): 1-25.

Fung, H. G., G. C. Lai, G. C. Patterson, and R. C. Witt, 1998, Underwriting Cycles in Property and Liability Insurance: An Empirical Analysis of Industry and By-Line Data, Journal of Risk and Insurance, 65(4): 539-562.

Genest, C., and L. Rivest, 1993, Statistical Inference Procedures for Bivariate Archimedean Copulas, Journal of the American Statistical Association, 88(423): 10341043.

Gerber, H. U., and E. S. Shiu, 1998, On the Time Value of Ruin, North American Actuarial Journal, 2(1): 48-72.

Glassermann, P., 2004, Monte Carlo Methods in Financial Engineering (New York: Springer).

Haugen, R. A., 1971, Insurer Risk Under Alternative Investment and Financing Strategies, Journal of Risk and Insurance, 38(1): 71-80.

Heilmann, W.-R., 1988, Fundamentals of Risk Theory (Karlsruhe: Verlag Versicherungswirtschaft).

Ibragimov, R., and J. Walden, 2007, The Limits of Diversification When Losses May Be Large, Journal of Banking and Finance, 31(8): 2551-2569. 
Ibragimov, R., D. Jaffee, and J. Walden, in press, Nondiversification Traps in Catastrophe Insurance Markets, Review of Financial Studies.

Joe, H., 1997, Multivariate Models and Dependence Concepts (London: Chapman \& Hall). Juri, A., and M. V. Wüthrich, 2002, Copula Convergence Theorems for Tail Events, Insurance: Mathematics and Economics, 30(3): 405-420.

Kahane, Y., and D. Nye, 1975, A Portfolio Approach to the Property-Liability Insurance Industry, Journal of Risk and Insurance, 42(7): 579-598.

Kaufmann, R., A. Gadmer, and R. Klett, 2001, Introduction to Dynamic Financial Analysis, ASTIN Bulletin, 31(1): 213-249.

Klugman, S. A., and R. Parsa, 1999, Fitting Bivariate Loss Distributions With Copulas, Insurance: Mathematics and Economics, 24(1): 139-148.

Kole, E., K. Koedijk, and M. Verbeek, 2007, Selecting Copulas for Risk Management, Journal of Banking \& Finance, 31(8): 2405-2423.

Lambert, E. W., and A. E. Hofflander, 1966, Impact of New Multiple Line Underwriting on Investment Portfolios of Property-Liability Insurers, Journal of Risk and Insurance, 33(2): 209-223.

Lamm-Tennant, J., and M. A. Weiss, 1997, International Insurance Cycles: Rational Expectations/Institutional Intervention. Journal of Risk and Insurance, 64(3): 415-439.

Li, S. X., and Z. Huang, 1996, Determination of the Portfolio Selection for a PropertyLiability Insurance Company, European Journal of Operational Research, 88, 257-268.

Lowe, S. P., and J. N. Stanard, 1997, An Integrated Dynamic Financial Analysis and Decision Support System for a Property Catastrophe Reinsurer, ASTIN Bulletin, 27(2): 339-371.

Luo, X., and P. V. Shevchenko, 2007, The $t$ Copula With Multiple Parameters of Degrees of Freedom: Bivariate Characteristics and Application to Risk Management, CMIS Report Number 2593, arXiv:0710.3959v1.

Malevergne, Y., and D. Sornette, 2003, Testing the Gaussian Copula Hypothesis for Financial Assets Dependences, Quantitative Finance, 3(4): 231-250.

Mashal, R., and A. Zeevi, 2002, Beyond Correlation: Extreme Co-Movements Between Financial Assets, Working Paper, Columbia Business School.

McNeil, A. J., 2007, Sampling Nested Archimedean Copulas, Journal of Statistical Computation and Simulation, 78(6): 567-581.

McNeil, A. J., R. Frey, and P. Embrechts, 2005, Quantitative Risk Management: Concepts, Techniques and Tools, (Princeton, NJ: Princeton University Press).

Meier, U. B., 2006, Existence and Causes of Insurance Cycles in Different Countries: Four Empirical Contributions. (Haupt, Bern: University of Zurich).

Nelsen, R. B., 2006, An Introduction to Copulas, 2nd edition (New York: Springer).

Niehaus, G., and A. Terry, 1993, Evidence on the Time Series Properties of Insurance Premiums and Causes of the Underwriting Cycle, Journal of Risk and Insurance, 60(3): 466-479.

Patton, A. J., 2006, Modelling Asymmetric Exchange Rate Dependence, International Economic Review, 47(2): 527-556. 
Pfeifer, D., and J. Nešlehová, 2004, Modeling and Generating Dependent Risk Processes for IRM and DFA, ASTIN Bulletin, 34(2): 333-360.

Powers, M. R., 1995, A Theory of Risk, Return and Solvency, Insurance: Mathematics and Economics, 17(2): 101-118.

Rolski, T., H. Schmidli, V. Schmidt, and J. Teugels, 1998, Stochastic Processes for Insurance and Finance (New York: Wiley).

Savu, C., and M. Trede, 2006, Hierarchical Archimedean Copulas, Working Paper, University of Münster.

Schmid, F., and R. Schmidt, 2007, Multivariate Extensions of Spearman's rho and Related Statistics, Statistics \& Probability Letters, 77(4): 407-416.

SCOR Switzerland AG, 2008, From Principle-Based Risk Management to Solvency Requirements. Swiss solvency test documentation, Zurich.

Sharpe, W. F., 1966, Mutual Fund Performance, Journal of Business, 39(1): 119-138.

Sun, J., E. W. Frees, and M. A. Rosenberg, 2008, Heavy-Tailed Longitudinal Data Modeling Using Copulas, Insurance: Mathematics and Economics, 42(2): 817-830.

Venezian, E. C., 1985, Ratemaking Methods and Profit Cycles in Property and Liability Insurance, Journal of Risk and Insurance, 52(3): 477-500.

Venter, G., 2002, Tails of Copulas, Proceedings of the Casualty Actuarial Society, 89(171): 68-113.

Wakker, P. P., R. H. Thaler, and A. Tversky, 1997, Probabilistic Insurance, Journal of Risk and Uncertainty, 15(1): 7-28.

Wang, S., 1998, Aggregation of Correlated Risk Portfolios: Models and Algorithms, Proceedings of the Casualty Actuarial Society, 85(163): 848-939.

Woo, G., 2002, Natural Catastrophes Probable Maximum Loss, British Actuarial Journal, 8(5): 943-959. 